Supplement of Earth Syst. Sci. Data, 11, 675-686, 2019

https://doi.org/10.5194/essd-11-675-2019-supplement

(c) Author(s) 2019. This work is distributed under

the Creative Commons Attribution 4.0 License.

(c) (1)

ज Earth System

¿ Science

亏Data

Supplement of

\title{
A leaf area index data set acquired in Sahelian rangelands of Gourma in Mali over the 2005-2017 period
}

Eric Mougin et al.

Correspondence to: Eric Mougin (eric.mougin@get.omp.eu)

The copyright of individual parts of the supplement might differ from the CC BY 4.0 License. 
Figure S1: Field photographs of the 4 monitored sites on sandy soils, taken during the dry (left) and the wet (right) seasons, from the same location, at the starting point of the sampling transect: a) Agoufou (\#17, Ag), b) Timbadior (\#18, Ti), c) Hombori Hondo (\#19, Ho), d) Tara (\#31, Ta).

a)

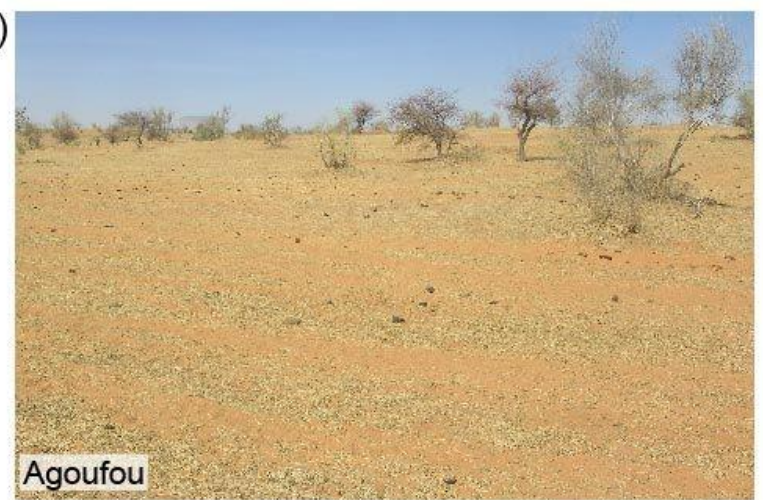

(2016/03/19)

b)

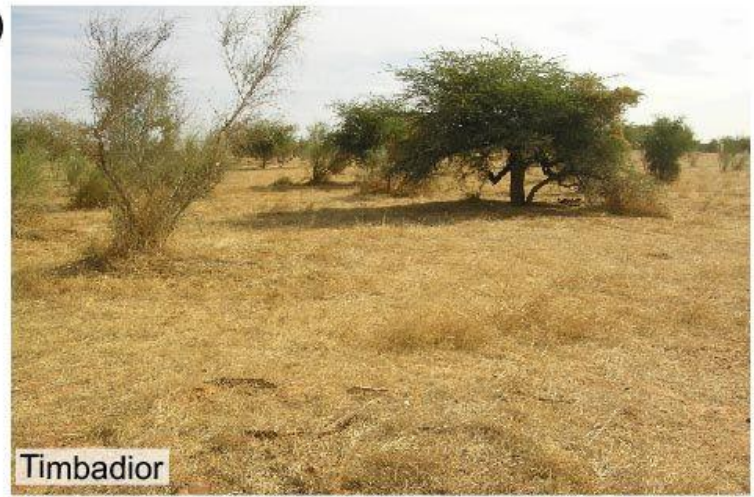

(2016/01/20)

c)

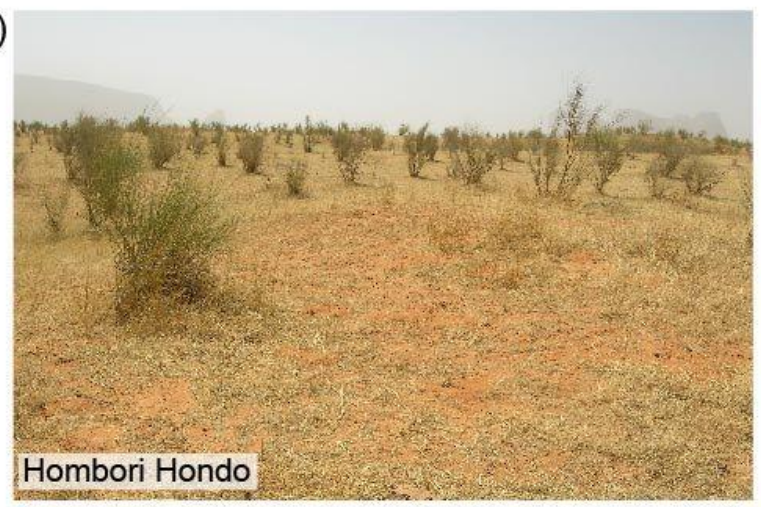

(2016/03/18)

d)

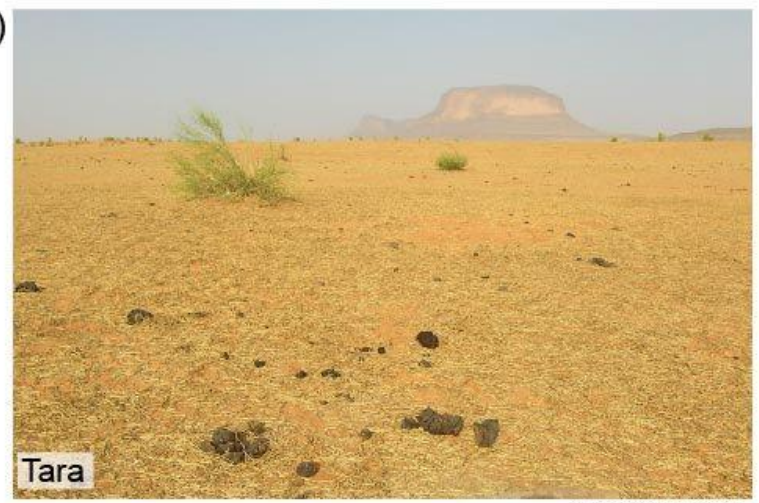

(2016/02/20)

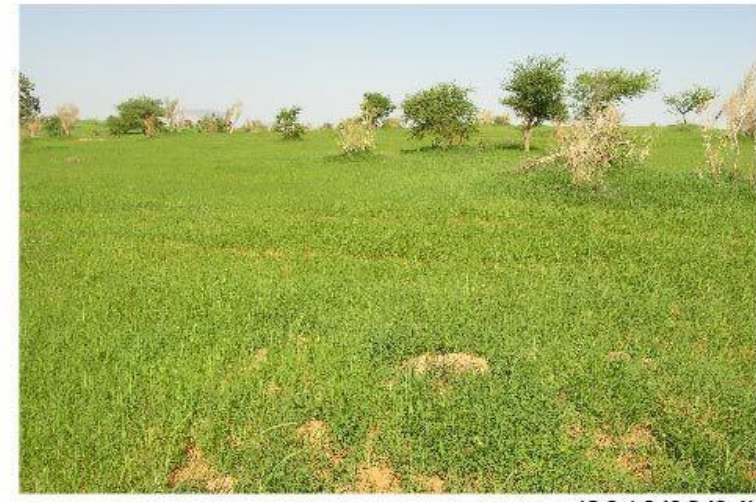

(2016/08/04)

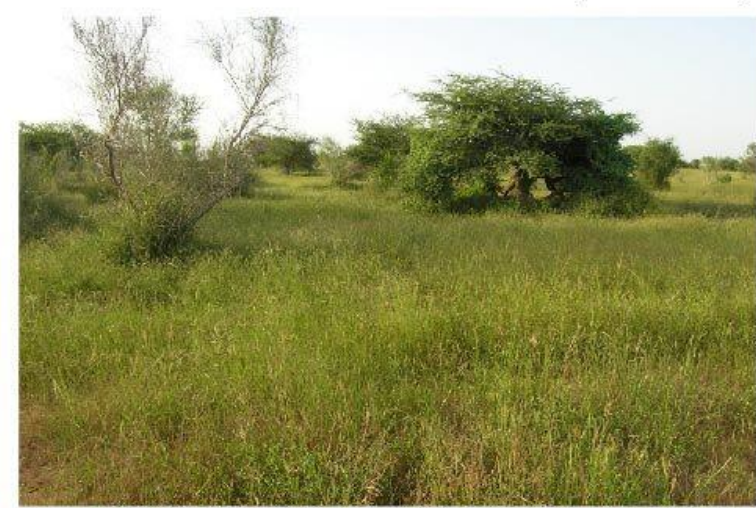

(2015/09/07)

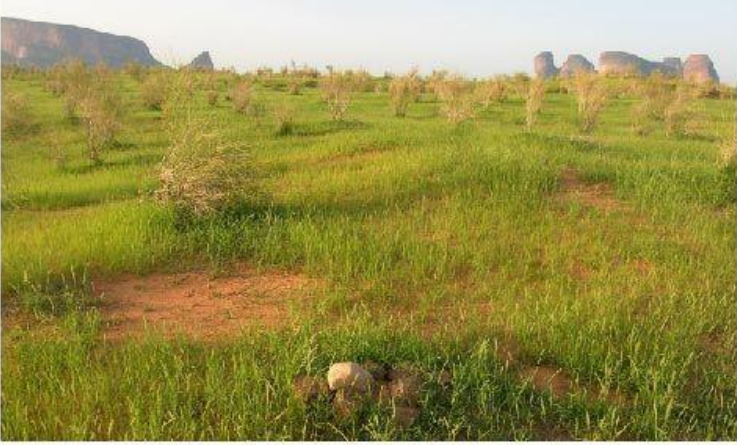

(2016/08/20)

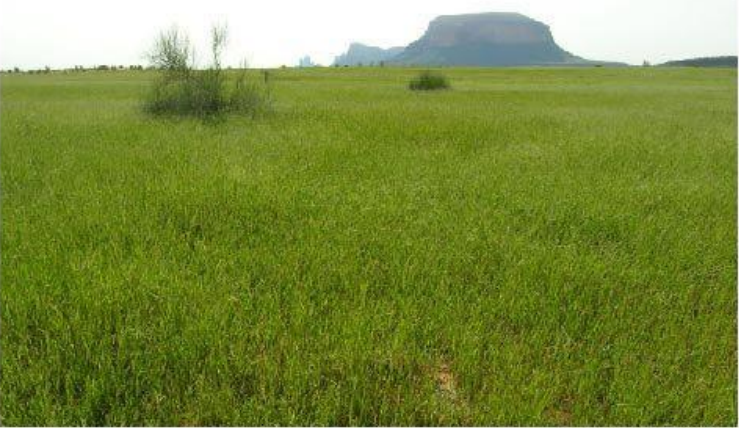


Figure S2: Field photographs of the monitored sites on loamy-clayed soils a) Kelma forest (\#21, Kf) and b) Kelma plain (\# 21b, Kp); on erosion surface c) Eguerit (\#40, Eg), and d) a millet field at Bilantao (\#41, Bi), taken during the dry (left) and the wet (right) seasons from the same location, at the starting point of the sampling transect.

a)

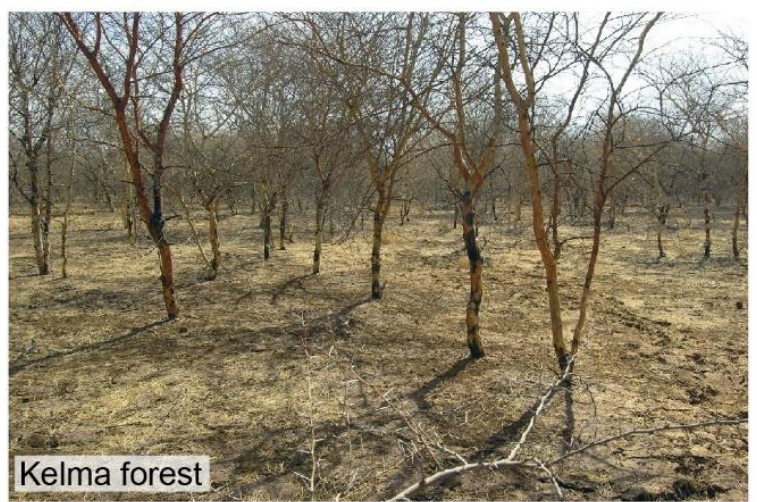

(2016/03/24)

b)

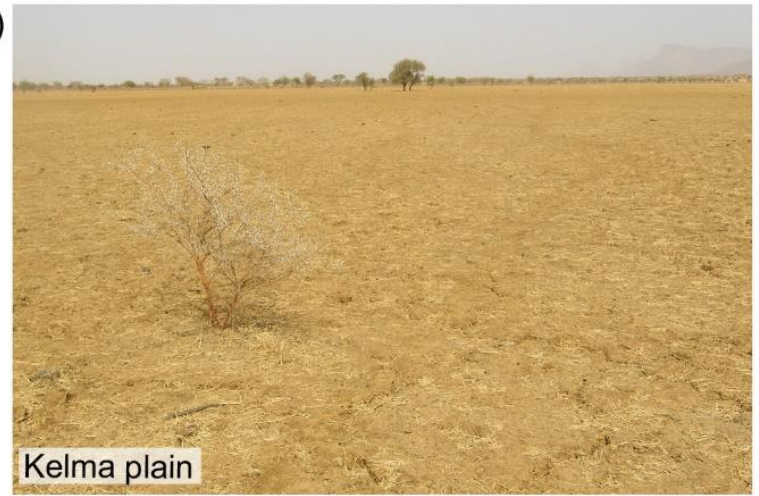

(2016/06/15)

c)

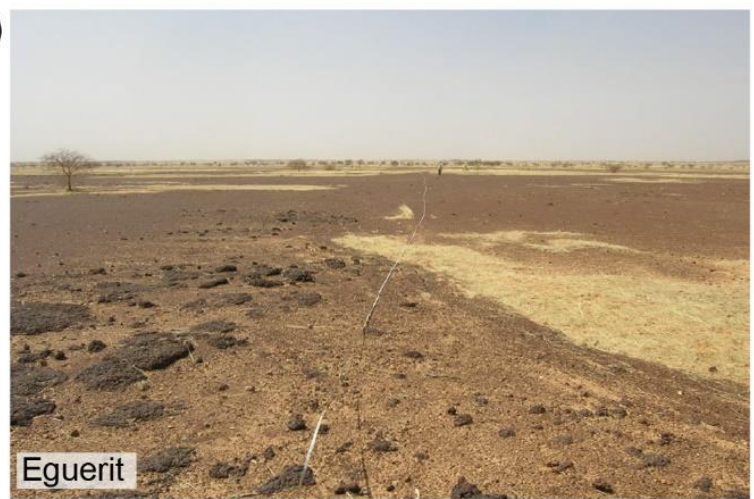

(2010/02/05)

d)

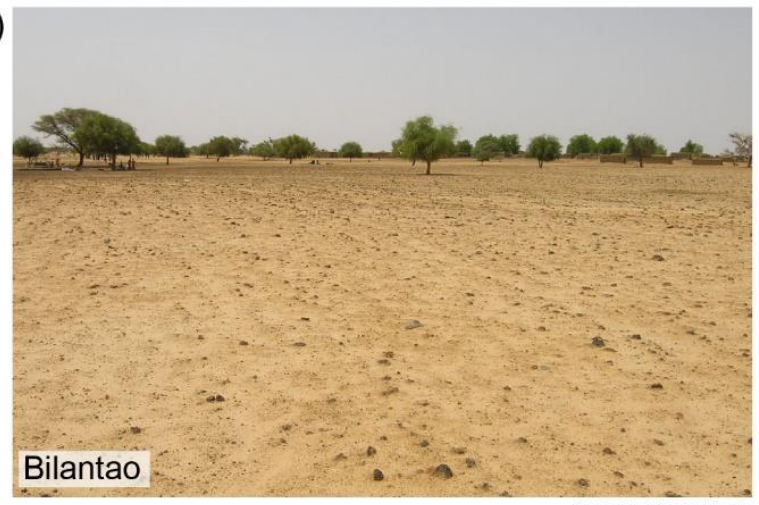

(2017/06/15)

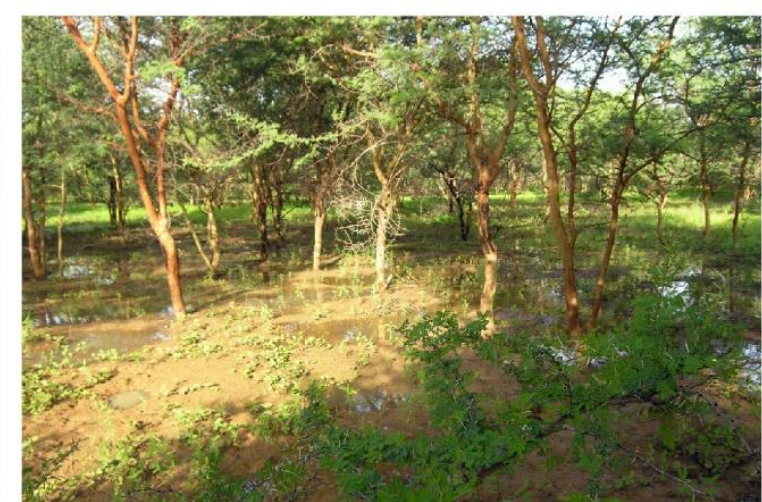

(2016/08/31)

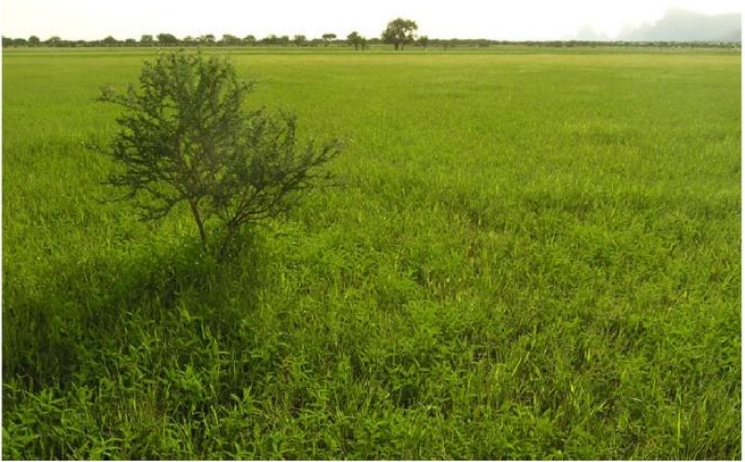

(2016/08/31)

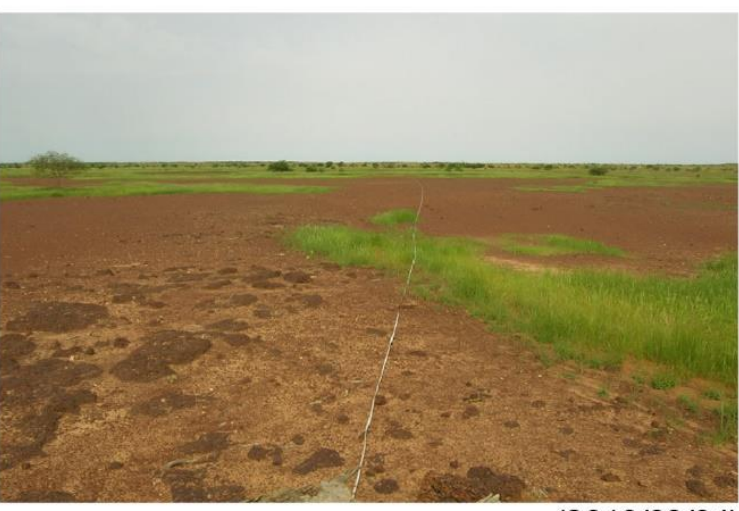

(2010/09/04)

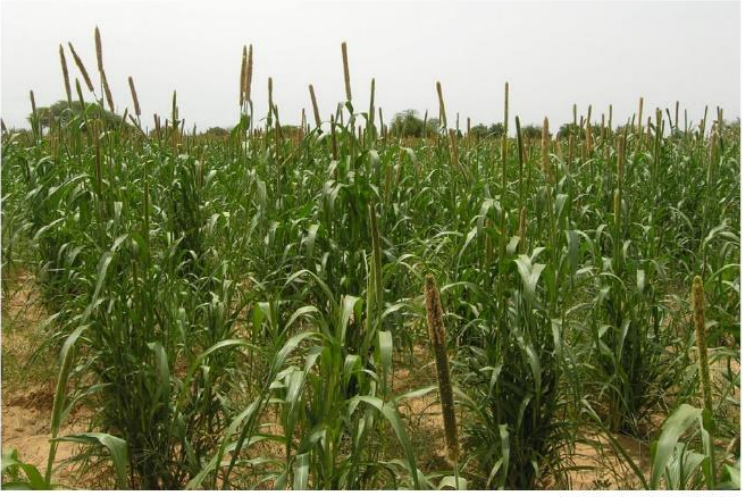

(2016/09/10) 
Table S1. General characteristics of the monitored sites.

\begin{tabular}{|c|c|c|c|c|c|}
\hline $\begin{array}{l}\text { Site name (code number }{ }^{\text {a }} \\
\text { Central coordinates } \\
\text { (longitude, latitude) }\end{array}$ & $\begin{array}{l}\text { Vegetation } \\
\text { type }\end{array}$ & $\begin{array}{l}\text { Dominant herbaceous } \\
\text { species }\end{array}$ & Dominant woody species & $\begin{array}{l}\text { Soil } \\
\text { type }\end{array}$ & $\begin{array}{l}\text { Grazing } \\
\text { pressure }\end{array}$ \\
\hline $\begin{array}{l}\text { Agoufou (\#17) } \\
\left(15.339^{\circ} \mathrm{N}, 1.484^{\circ} \mathrm{W}\right)\end{array}$ & $\begin{array}{l}\text { Open woody } \\
\text { savannah }\end{array}$ & $\begin{array}{l}\text { Cenchrus biflorus, } \\
\text { Aristida mutabilis, } \\
\text { Zornia glochidiata, } \\
\text { Tragus berteroniamus }\end{array}$ & $\begin{array}{l}\text { Acacia raddiana, } \\
\text { Combretum glutinosum, } \\
\text { Balanites aegyptiaca, } \\
\text { Acacia senegal, } \\
\text { Leptadenia pyrotechnica }\end{array}$ & Sand & High \\
\hline $\begin{array}{l}\text { Eguerit }(\# 40) \\
\left(15.504^{\circ} \mathrm{N}, 1.397^{\circ} \mathrm{W}\right)\end{array}$ & $\begin{array}{l}\text { Scattered } \\
\text { shrubs, herbs } \\
\text { on loamy } \\
\text { deposits }\end{array}$ & $\begin{array}{l}\text { Schoenefeldia gracilis, } \\
\text { Aristida adscentionis, } \\
\text { Pennisetum violaceum, } \\
\text { Panicum laetum }\end{array}$ & $\begin{array}{l}\text { Acacia ehrenbergiana, } \\
\text { Commiphora Africana, } \\
\text { Maerua crassifolia, } \\
\text { Ziziphus mauritiana }\end{array}$ & $\begin{array}{l}\text { Gravels } \\
\text { and } \\
\text { loamy } \\
\text { patches }\end{array}$ & Low \\
\hline $\begin{array}{l}\text { Timbadior }(\# 18) \\
\left(15.332^{\circ} \mathrm{N}, 1.546^{\circ} \mathrm{W}\right)\end{array}$ & $\begin{array}{l}\text { Open woody } \\
\text { savannah }\end{array}$ & $\begin{array}{l}\text { Cenchrus biflorus, } \\
\text { Aristida mutabilis, } \\
\text { Zornia glochidiata, } \\
\text { Tragus berteroniamus }\end{array}$ & $\begin{array}{l}\text { Acacia raddiana, } \\
\text { Combretum glutinosum, } \\
\text { Balanites aegyptiaca, } \\
\text { Acacia senegal, } \\
\text { Leptadenia pyrotechnica }\end{array}$ & Sand & Moderate \\
\hline $\begin{array}{l}\text { Hombori Hondo (\#19) } \\
\left(15.322^{\circ} \mathrm{N}, 1.698^{\circ} \mathrm{W}\right)\end{array}$ & $\begin{array}{l}\text { Open shrub } \\
\text { savannah }\end{array}$ & $\begin{array}{l}\text { Aristida sieberiana, } \\
\text { Chrozophora } \\
\text { senegalensis, } \\
\text { Heliotropium strigosum }\end{array}$ & $\begin{array}{l}\text { Balanites aegyptiaca, } \\
\text { Leptadenia pyrotechnica }\end{array}$ & Sand & High \\
\hline $\begin{array}{l}\text { Kelma Forest (\#21) } \\
\text { and } \\
\text { Kelma Herbs }(\# 21) \\
15.219^{\circ} \mathrm{N}, 1.566^{\circ} \mathrm{W}\end{array}$ & $\begin{array}{l}\text { Seasonally } \\
\text { inundated } \\
\text { open Acacia } \\
\text { forest }\end{array}$ & $\begin{array}{l}\text { Aeschinomene sensitive, } \\
\text { Echinocloa colona, } \\
\text { Sporobolus hevolvus }\end{array}$ & $\begin{array}{l}\text { Acacia seyal, } \\
\text { Acacia nilotica, } \\
\text { Balanites aegyptiaca }\end{array}$ & $\begin{array}{l}\text { Clay, } \\
\text { loam }\end{array}$ & High \\
\hline $\begin{array}{l}\text { Kelma Plain (\#21b) } \\
\left(15.219^{\circ} \mathrm{N}, 1.573^{\circ} \mathrm{W}\right)\end{array}$ & $\begin{array}{l}\text { Seasonally } \\
\text { inundated } \\
\text { plain }\end{array}$ & $\begin{array}{l}\text { Aeschinomene sensitive, } \\
\text { Echinocloa colona, } \\
\text { Panicum laetum, } \\
\text { Sporobolus hevolvus }\end{array}$ & $\begin{array}{l}\text { Acacia seyal, } \\
\text { Balanites aegyptiaca }\end{array}$ & $\begin{array}{l}\text { Silt, } \\
\text { loam }\end{array}$ & High \\
\hline $\begin{array}{l}\text { Tara }(\# 31) \\
\left(15.230^{\circ} \mathrm{N}, 1.583^{\circ} \mathrm{W}\right)\end{array}$ & $\begin{array}{l}\text { Open woody } \\
\text { savannah }\end{array}$ & $\begin{array}{l}\text { Cenchrus biflorus, } \\
\text { Aristida sieberiana, } \\
\text { Dactyloctenium } \\
\text { aegyptium, } \\
\text { Zornia glochidiata, } \\
\text { Tragus berteroniamus, } \\
\text { Schoenefeldia gracilis }\end{array}$ & $\begin{array}{l}\text { Acacia raddiana, } \\
\text { Balanites aegyptiaca, } \\
\text { Combretum glutinosum, } \\
\text { Leptadenia pyrotechnica }\end{array}$ & Sand & High \\
\hline $\begin{array}{l}\text { Bilantao (\#41) } \\
\left(15.285^{\circ} \mathrm{N}, 1.558^{\circ} \mathrm{W}\right)\end{array}$ & $\begin{array}{l}\text { Millet field } \\
\text { (Pennisetum } \\
\text { glaucum) }\end{array}$ & Pennisetum glaucum & $\begin{array}{l}\text { Acacia raddiana, } \\
\text { Balanites aegyptiaca, } \\
\text { Faidherbia albida }\end{array}$ & Sand & Low \\
\hline
\end{tabular}

${ }^{a}$ Site code number refers to Hiernaux et al. (2009a) 
Table S2. Top soil texture data in terms of sand, clay and silt contents (\%) for the monitored sites: Agoufou, Bilantao, Eguerit, Hombori Hondo, Kelma forest, Kelma plain, Tara and Timbadior. Particles size are defined as clay $(<0.002 \mathrm{~mm})$, silt $(<0.05 \mathrm{~mm})$, and sand $(<2 \mathrm{~mm})$. Soil texture data were taken from Hiernaux et al. (1984), except for Bilantao, Kelma plain and Tara which soil texture was analysed in 2018 by the Laboratoire Sol-Eau-Plantes (Institut d'Economie Rurale, Sotuba, Mali).

\begin{tabular}{|c|c|c|c|c|c|c|c|c|c|c|c|c|}
\hline \multirow{2}{*}{$\begin{array}{l}\text { Depth } \\
\text { (cm) }\end{array}$} & \multicolumn{3}{|c|}{$\begin{array}{c}\left.\text { Agoufou (\#17 }{ }^{\mathrm{a}}\right) \\
\left(15.339^{\circ} \mathrm{N}, \mathbf{1 . 4 8 4}^{\circ} \mathrm{W}\right)\end{array}$} & \multicolumn{3}{|c|}{$\begin{array}{l}\text { Bilantao (\#41) } \\
\left(15 .^{\circ} \mathrm{N}, 1.4^{\circ} \mathrm{W}\right)\end{array}$} & \multicolumn{3}{|c|}{$\begin{array}{c}\text { Eguerit }(\# 40) \\
\left(15.50^{\circ} \mathrm{N}, 1.40^{\circ} \mathrm{W}\right)\end{array}$} & \multicolumn{3}{|c|}{$\begin{array}{c}\text { Kelma Forest }(\# 21) \\
\left(15.219^{\circ} \mathrm{N}, \mathbf{1 . 5 6 6}^{\circ} \mathrm{W}\right)\end{array}$} \\
\hline & Sand & Silt & Clay & Sand & Silt & Clay & Sand & Silt & Clay & Sand & Silt & Clay \\
\hline $0-6$ & 91.0 & 3.3 & 4.6 & 96.9 & 1.0 & 2.1 & 43.6 & 29.8 & 26.3 & 47.8 & 27.5 & 24.5 \\
\hline $6-12$ & 91.1 & 3.2 & 5.1 & 97.4 & 1.3 & 1.3 & 43.6 & 27.9 & 28.1 & 40.3 & 19.4 & 40.0 \\
\hline $12-25$ & 89.7 & 4.1 & 5.8 & 96.8 & 1.6 & 1.6 & 30.2 & 27.4 & 42.1 & 35.1 & 19.8 & 45.1 \\
\hline $25-50$ & 90.9 & 3.2 & 5.5 & 93.5 & 4.9 & 1.6 & - & - & - & 31.6 & 16.1 & 52.4 \\
\hline $50-100$ & 92.0 & 1.9 & 5.8 & 87.1 & 9.7 & 3.2 & - & - & - & 30.1 & 21 & 48.9 \\
\hline
\end{tabular}

\begin{tabular}{|c|c|c|c|c|c|c|c|c|c|c|c|c|}
\hline \multirow{2}{*}{$\begin{array}{c}\text { Depth } \\
\text { (cm) }\end{array}$} & \multicolumn{3}{|c|}{$\begin{array}{l}\text { Kelma Plain (\#21b) } \\
\left(15.219^{\circ} \mathrm{N}, 1^{\left.1.573^{\circ} \mathrm{W}\right)}\right.\end{array}$} & \multicolumn{3}{|c|}{$\begin{array}{l}\text { Hombori Hondo (\#19) } \\
\left(15.50^{\circ} \mathrm{N}, 1.40^{\circ} \mathrm{W}\right)\end{array}$} & \multicolumn{3}{|c|}{$\begin{array}{c}\text { Tara }(\# 31) \\
\left(15.219^{\circ} \mathrm{N}, 1.566^{\circ} \mathrm{W}\right)\end{array}$} & \multicolumn{3}{|c|}{$\begin{array}{c}\text { Timbadior (\#18) } \\
\left(15.339^{\circ} \mathrm{N}, \mathbf{1 . 4 8 4}^{\circ} \mathrm{W}\right)\end{array}$} \\
\hline & Sand & Silt & Clay & Sand & Silt & Clay & Sand & Silt & Clay & Sand & Silt & Clay \\
\hline $0-6$ & 50 & 49 & 1 & 90.5 & 1.1 & 6.7 & 94.0 & 2.0 & 4.0 & 92.0 & 2.8 & 4.9 \\
\hline 6-12 & 20 & 78 & 2 & 94.1 & 1.4 & 3.6 & 94.0 & 2.0 & 4.0 & 89.9 & 4.4 & 5.2 \\
\hline $12-25$ & 20 & 78 & 2 & 93.1 & 3.4 & 3.4 & 96.0 & 2.0 & 2.0 & 89.5 & 5.3 & 4.9 \\
\hline $25-50$ & 19 & 79 & 2 & 92.9 & 2.6 & 4.3 & 96.0 & 2.0 & 2.0 & 90.5 & 2.9 & 5.8 \\
\hline $50-100$ & 31 & 67 & 2 & 92.9 & 2.6 & 4.3 & 96.0 & 2.0 & 2.0 & 91.3 & 2.8 & 5.8 \\
\hline
\end{tabular}

${ }^{a}$ Site code number refers to Hiernaux et al. (2009a). 
Table S3. Coordinates (latitude, longitude) of the starting, centre and ending points of the sampling transects. The directions of the transects are indicated by NS (North-South), EW (East-West), NE (North-East) and SW (South-West).

\begin{tabular}{|c|c|c|c|}
\hline Site $(\#$, transect) & Starting Point $\left({ }^{\circ} \mathbf{N},{ }^{\circ} \mathbf{W}\right)$ & Centre point $^{\left({ }^{\circ} \mathbf{N},{ }^{\circ} \mathbf{W}\right)}$ & Ending Point $\left({ }^{\circ} \mathbf{N},{ }^{\circ} \mathbf{W}\right)$ \\
\hline Agoufou NS (\#17) & $15.34347,1.48475$ & $15.33927,1.48413$ & 15.33447 .1 .48320 \\
\hline Agoufou EW (\#17) & $15.34005,1.47950$ & $15.33927,1.48413$ & 15.33952 .1 .48885 \\
\hline Bilantao NE-SW (\#41) & $15.28956,1.55867$ & $15.28524,1.55867$ & 15.28091 .1 .55867 \\
\hline Eguerit EW (\#40) & $15.50522,1.39240$ & $15.50409,1.39756$ & 15.50297 .1 .40272 \\
\hline Hombori_Hondo NS (\#19) & $15.32692,1.69835$ & $15.32240,1.69828$ & 15.31788 .1 .69821 \\
\hline Hombori Hondo EW (\#19) & $15.32222,1.69333$ & $15.32240,1.69828$ & $15.32177,1.70277$ \\
\hline Kelma Forest EW (\#21) & $15.21877,1.56806$ & $15.21887,1.56574$ & 15.21897 .1 .56342 \\
\hline Kelma Herbs EW (\#21) & $15.21877,1.56806$ & $15.21887,1.56690$ & $15.21887,1.56574$ \\
\hline Kelma Plain EW (\#21b) & $15.21777,1.56972$ & $15.21749,1.57154$ & $15.21722,1.57333$ \\
\hline Tara NE-SW (\#31) & $15.23260,1.57943$ & $15.23013,1.58335$ & 15.22824 .1 .58757 \\
\hline Tara NW-SE (\#31) & $15.22985,1.57872$ & $15.23013,1.58335$ & 15.23008 .1 .58802 \\
\hline Timbadior NS (\#18) & $15.33680,1.54683$ & $15.33231,1.54627$ & 15.32782 .1 .54570 \\
\hline Timbadior EW (\#18) & $15.33382,1.54187$ & $15.33231,1.54627$ & 15.33091 .1 .55067 \\
\hline
\end{tabular}

${ }^{a}$ Site code number refers to Hiernaux et al. (2009a) 
Table S4. Dates of hemispheric photograph acquisition over the 2005-2017 period.

\begin{tabular}{|c|c|c|c|c|c|c|c|}
\hline Agoufou EW (\#17) & 2005 & 2006 & 2007 & 2008 & 2009 & 2010 & 2011 \\
\hline & $2005 / 06 / 06$ & $2006 / 07 / 14$ & $2007 / 07 / 16$ & $2008 / 07 / 05$ & $2009 / 07 / 06$ & $2010 / 07 / 06$ & $2011 / 07 / 08$ \\
\hline & $2005 / 06 / 17$ & $2006 / 07 / 29$ & $2007 / 07 / 26$ & $2008 / 07 / 15$ & 2009/07/16 & $2010 / 08 / 05$ & $2011 / 07 / 14$ \\
\hline & $2005 / 06 / 24$ & $2006 / 08 / 10$ & $2007 / 07 / 29$ & $2008 / 07 / 25$ & $2009 / 07 / 28$ & $2010 / 08 / 17$ & $2011 / 07 / 18$ \\
\hline & $2005 / 07 / 04$ & $2006 / 08 / 20$ & $2007 / 07 / 30$ & 2008/08/08 & 2009/08/06 & 2010/09/03 & $2011 / 07 / 24$ \\
\hline & $2005 / 07 / 17$ & $2006 / 08 / 29$ & $2007 / 08 / 05$ & $2008 / 08 / 14$ & 2009/08/16 & 2010/09/04 & $2011 / 08 / 10$ \\
\hline & $2005 / 07 / 24$ & $2006 / 09 / 10$ & $2007 / 08 / 13$ & $2008 / 08 / 18$ & $2009 / 08 / 26$ & $2010 / 09 / 13$ & $2011 / 08 / 21$ \\
\hline & $2005 / 07 / 30$ & $2006 / 09 / 20$ & $2007 / 08 / 14$ & $2008 / 08 / 24$ & $2009 / 08 / 31$ & $2010 / 09 / 30$ & $2011 / 08 / 26$ \\
\hline & $2005 / 08 / 03$ & $2006 / 09 / 30$ & $2007 / 08 / 23$ & $2008 / 08 / 31$ & 2009/09/03 & & 2011/09/03 \\
\hline & $2005 / 08 / 13$ & & $2007 / 08 / 27$ & 2008/09/07 & 2009/09/05 & & 2011/09/09 \\
\hline & $2005 / 08 / 20$ & & 2007/09/06 & $2008 / 09 / 20$ & 2009/09/15 & & \\
\hline & $2005 / 08 / 24$ & & $2007 / 09 / 16$ & & & & \\
\hline & $2005 / 08 / 28$ & & $2007 / 09 / 26$ & & & & \\
\hline & $2005 / 09 / 28$ & & & & & & \\
\hline & 2012 & 2013 & 2014 & 2015 & 2016 & 2017 & \\
\hline & $2012 / 06 / 26$ & $2013 / 06 / 05$ & $2014 / 07 / 05$ & $2015 / 07 / 11$ & $2016 / 06 / 24$ & $2017 / 0715$ & \\
\hline & $2012 / 07 / 10$ & 2013/07/07 & $2014 / 08 / 16$ & $2015 / 07 / 20$ & 2016/07/08 & $2017 / 07 / 23$ & \\
\hline & $2012 / 07 / 24$ & $2013 / 07 / 16$ & $2014 / 08 / 27$ & $2015 / 07 / 30$ & $2016 / 07 / 18$ & $2017 / 08 / 03$ & \\
\hline & 2012/08/04 & $2013 / 07 / 31$ & $2014 / 09 / 05$ & $2015 / 08 / 10$ & $2016 / 07 / 28$ & $2017 / 08 / 13$ & \\
\hline & $2012 / 08 / 14$ & $2013 / 08 / 19$ & $2014 / 09 / 15$ & $2015 / 08 / 20$ & 2016/08/11 & $2017 / 08 / 22$ & \\
\hline & $2012 / 08 / 25$ & $2013 / 08 / 29$ & $2014 / 09 / 25$ & $2015 / 08 / 29$ & $2016 / 08 / 27$ & $2017 / 09 / 03$ & \\
\hline & 2012/09/04 & 2013/09/09 & & 2015/09/09 & $2016 / 09 / 10$ & $2017 / 09 / 14$ & \\
\hline & $2012 / 09 / 18$ & 2013/09/19 & & $2015 / 09 / 16$ & $2016 / 09 / 28$ & $2017 / 09 / 25$ & \\
\hline & $2012 / 09 / 29$ & $2013 / 09 / 29$ & & $2015 / 09 / 27$ & & & \\
\hline & & 2013/10/09 & & & & & \\
\hline Agoufou NS (\#17) & 2005 & 2006 & 2007 & 2008 & 2009 & 2010 & 2011 \\
\hline
\end{tabular}




\begin{tabular}{|c|c|c|c|c|c|c|c|}
\hline & - & - & $2007 / 07 / 30$ & $2008 / 07 / 25$ & $2009 / 07 / 06$ & $2010 / 07 / 06$ & $2011 / 07 / 18$ \\
\hline & & & 2007/08/14 & 2008/08/18 & $2009 / 07 / 16$ & & 2011/07/24 \\
\hline & & & $2007 / 08 / 27$ & $2008 / 08 / 24$ & $2009 / 07 / 28$ & & $2011 / 08 / 10$ \\
\hline & & & & $2008 / 08 / 31$ & 2009/08/06 & & $2011 / 08 / 21$ \\
\hline & & & & 2008/09/07 & $2009 / 08 / 16$ & & $2011 / 08 / 26$ \\
\hline & & & & & $2009 / 08 / 26$ & & 2011/09/09 \\
\hline & & & & & $2009 / 08 / 31$ & & \\
\hline & & & & & 2009/09/05 & & \\
\hline & 2012 & 2013 & 2014 & 2015 & 2016 & 2017 & \\
\hline & - & - & - & - & - & - & \\
\hline Timbadior EW (\#18) & 2005 & 2006 & 2007 & 2008 & 2009 & 2010 & 2011 \\
\hline & - & - & $2007 / 08 / 01$ & $2008 / 08 / 12$ & $2009 / 07 / 11$ & - & - \\
\hline & & & 2007/08/16 & 2008/08/18 & $2009 / 07 / 30$ & & \\
\hline & & & & $2008 / 08 / 23$ & 2009/08/08 & & \\
\hline & & & & 2008/09/02 & $2009 / 08 / 27$ & & \\
\hline & 2012 & 2013 & 2014 & 2015 & 2016 & 2017 & \\
\hline & - & - & - & - & - & - & \\
\hline Timbadior NS (\#18) & 2005 & 2006 & 2007 & 2008 & 2009 & 2010 & 2011 \\
\hline & & & $2007 / 07 / 18$ & $2008 / 07 / 22$ & $2009 / 07 / 11$ & $2010 / 07 / 05$ & $2011 / 07 / 18$ \\
\hline & & & 2007/07/30 & $2008 / 07 / 26$ & $2009 / 07 / 30$ & 2010/08/05 & $2011 / 08 / 10$ \\
\hline & & & 2007/08/01 & 2008/08/12 & 2009/08/08 & & $2011 / 08 / 21$ \\
\hline & & & 2007/08/16 & 2008/08/18 & $2009 / 08 / 18$ & & 2011/09/09 \\
\hline & & & 2007/09/08 & 2008/09/02 & $2009 / 08 / 27$ & & \\
\hline & 2012 & 2013 & 2014 & 2015 & 2016 & 2017 & \\
\hline & - & - & $2014 / 08 / 27$ & - & - & - & \\
\hline Hombori Hondo EW & 2005 & 2006 & 2007 & 2008 & 2009 & 2010 & 2011 \\
\hline (\#19) & - & - & 2007/08/01 & 2008/08/12 & $2009 / 07 / 05$ & - & - \\
\hline & & & 2007/08/16 & 2008/08/24 & $2009 / 07 / 30$ & & \\
\hline & & & & 2008/09/04 & 2009/08/08 & & \\
\hline & & & & & $2009 / 08 / 28$ & & \\
\hline
\end{tabular}




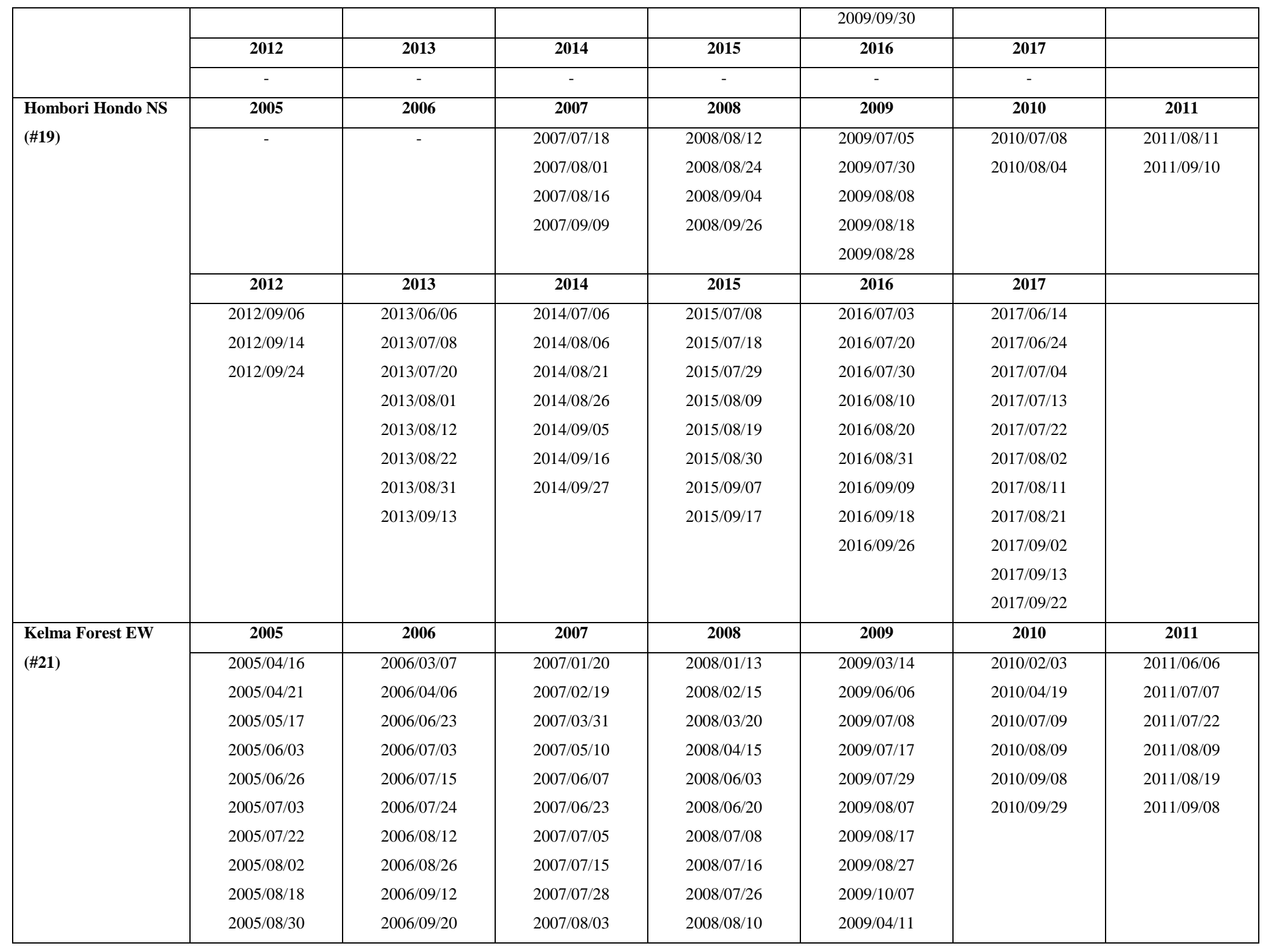




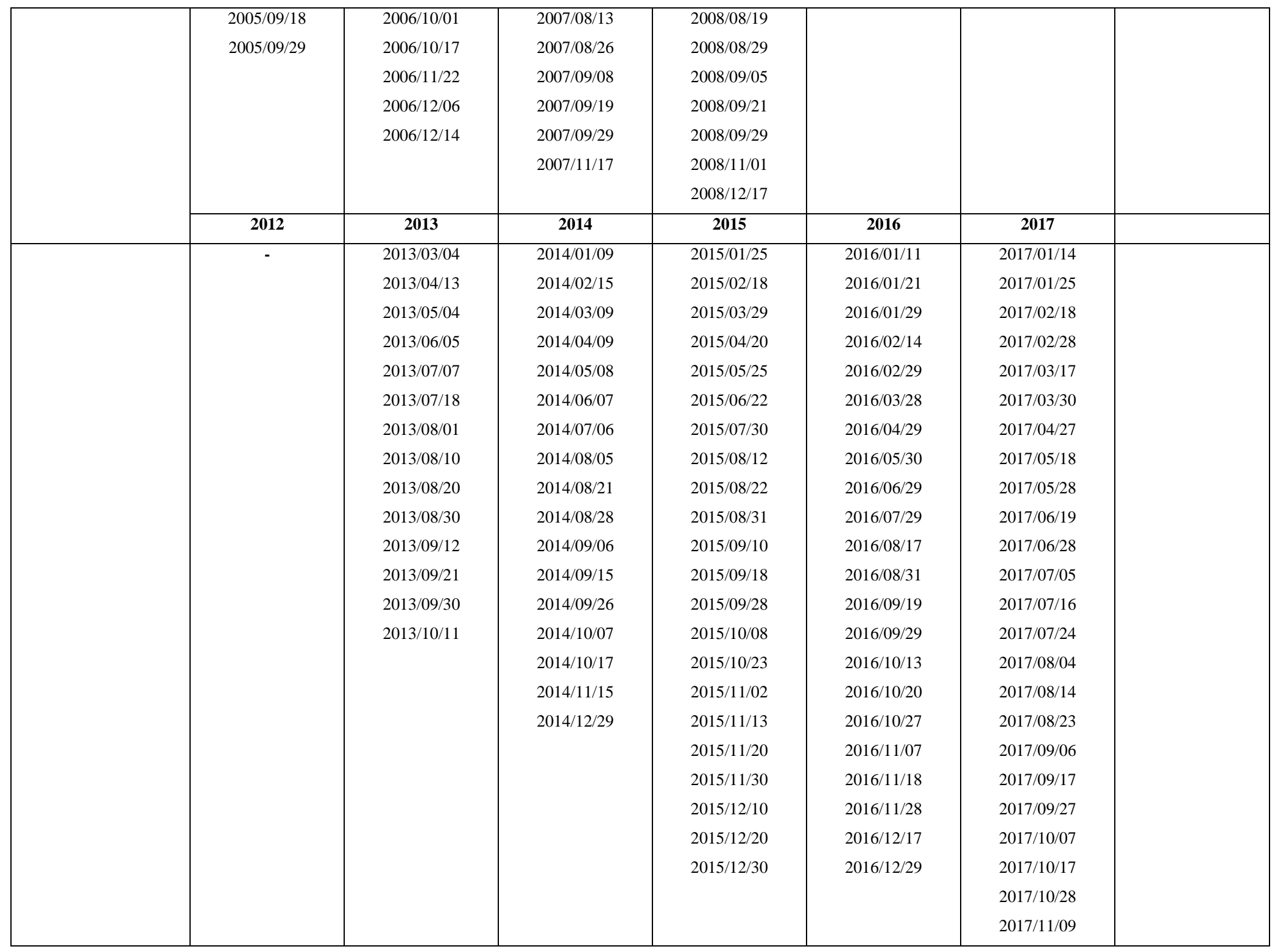




\begin{tabular}{|c|c|c|c|c|c|c|c|}
\hline & & & & & & $\begin{array}{l}2017 / 11 / 19 \\
2017 / 11 / 29 \\
2017 / 12 / 08 \\
2017 / 12 / 17 \\
2017 / 12 / 30\end{array}$ & \\
\hline \multirow{4}{*}{$\begin{array}{l}\text { Kelma Herbs EW } \\
(\# 21)\end{array}$} & 2005 & 2006 & 2007 & 2008 & 2009 & 2010 & 2011 \\
\hline & $\begin{array}{l}2005 / 06 / 26 \\
2005 / 07 / 03 \\
2005 / 07 / 22 \\
2005 / 08 / 18 \\
2005 / 08 / 30 \\
2005 / 09 / 18\end{array}$ & $\begin{array}{l}2006 / 07 / 24 \\
2006 / 08 / 12 \\
2006 / 08 / 26 \\
2006 / 09 / 12 \\
2006 / 09 / 20 \\
2006 / 10 / 01 \\
2006 / 10 / 17 \\
2006 / 11 / 22\end{array}$ & $\begin{array}{l}2007 / 07 / 15 \\
2007 / 07 / 28 \\
2007 / 08 / 03 \\
2007 / 08 / 13 \\
2007 / 08 / 26 \\
2007 / 09 / 08 \\
2007 / 09 / 19 \\
2007 / 09 / 29 \\
2007 / 11 / 17\end{array}$ & $\begin{array}{l}2008 / 07 / 08 \\
2008 / 07 / 16 \\
2008 / 07 / 26 \\
2008 / 08 / 10 \\
2008 / 08 / 19 \\
2008 / 08 / 29 \\
2008 / 09 / 05 \\
2008 / 09 / 21 \\
2008 / 09 / 29\end{array}$ & $\begin{array}{l}2009 / 08 / 17 \\
2009 / 08 / 27 \\
2009 / 10 / 07 \\
2009 / 11 / 04\end{array}$ & $\begin{array}{l}2010 / 07 / 09 \\
2010 / 08 / 09\end{array}$ & $\begin{array}{l}2011 / 07 / 22 \\
2011 / 08 / 09 \\
2011 / 08 / 19\end{array}$ \\
\hline & 2012 & 2013 & 2014 & 2015 & 2016 & 2017 & \\
\hline & $\begin{array}{l}2012 / 07 / 25 \\
2012 / 08 / 06 \\
2012 / 08 / 17 \\
2012 / 08 / 26 \\
2012 / 09 / 07 \\
2012 / 09 / 19 \\
2012 / 09 / 30 \\
2012 / 10 / 13 \\
2012 / 10 / 24 \\
2012 / 10 / 30 \\
2012 / 11 / 10\end{array}$ & $\begin{array}{l}2013 / 08 / 30 \\
2013 / 09 / 12 \\
2013 / 09 / 21 \\
2013 / 09 / 30 \\
2013 / 10 / 11\end{array}$ & $\begin{array}{l}2014 / 08 / 21 \\
2014 / 08 / 28 \\
2014 / 09 / 06 \\
2014 / 09 / 15 \\
2014 / 09 / 26 \\
2014 / 10 / 07 \\
2014 / 10 / 17\end{array}$ & $\begin{array}{l}2015 / 07 / 20 \\
2015 / 07 / 30 \\
2015 / 08 / 12 \\
2015 / 08 / 22 \\
2015 / 08 / 31 \\
2015 / 09 / 10 \\
2015 / 09 / 18 \\
2015 / 09 / 28 \\
2015 / 10 / 08 \\
2015 / 10 / 23 \\
2015 / 11 / 02\end{array}$ & $\begin{array}{l}2016 / 08 / 17 \\
2016 / 08 / 31 \\
2016 / 09 / 19 \\
2016 / 09 / 29 \\
2016 / 10 / 13 \\
2016 / 10 / 20 \\
2016 / 10 / 27 \\
2016 / 11 / 07 \\
2016 / 11 / 18 \\
2016 / 11 / 28\end{array}$ & $\begin{array}{l}2017 / 08 / 04 \\
2017 / 08 / 14 \\
2017 / 08 / 23 \\
2017 / 09 / 06 \\
2017 / 09 / 17 \\
2017 / 09 / 27 \\
2017 / 10 / 07 \\
2017 / 10 / 17 \\
2017 / 10 / 28\end{array}$ & \\
\hline $\begin{array}{l}\text { Kelma Plain EW } \\
(\# 21 b)\end{array}$ & $\begin{array}{c}2005 \\
-\end{array}$ & $\begin{array}{c}2006 \\
-\end{array}$ & $\begin{array}{c}2007 \\
-\end{array}$ & $\begin{array}{c}\mathbf{2 0 0 8} \\
2008 / 08 / 10\end{array}$ & $\begin{array}{c}\mathbf{2 0 0 9} \\
2009 / 08 / 07 \\
2009 / 08 / 17 \\
2009 / 08 / 27 \\
2009 / 09 / 16\end{array}$ & $\begin{array}{c}2010 \\
-\end{array}$ & $\begin{array}{c}\mathbf{2 0 1 1} \\
2011 / 07 / 22 \\
2011 / 08 / 09 \\
2011 / 08 / 19\end{array}$ \\
\hline
\end{tabular}




\begin{tabular}{|c|c|c|c|c|c|c|c|}
\hline & & & & & $2009 / 10 / 07$ & & \\
\hline & 2012 & 2013 & 2014 & 2015 & 2016 & 2017 & \\
\hline & $2012 / 06 / 17$ & $2013 / 06 / 05$ & $2014 / 08 / 05$ & $2015 / 07 / 11$ & $2016 / 07 / 20$ & $2017 / 06 / 19$ & \\
\hline & $2012 / 06 / 30$ & $2013 / 07 / 07$ & $2014 / 08 / 21$ & $2015 / 07 / 20$ & $2016 / 07 / 29$ & $2017 / 06 / 28$ & \\
\hline & $2012 / 07 / 07$ & 2013/07/18 & $2014 / 08 / 28$ & $2015 / 07 / 30$ & $2016 / 08 / 10$ & $2017 / 07 / 05$ & \\
\hline & $2012 / 07 / 25$ & $2013 / 08 / 01$ & $2014 / 09 / 06$ & $2015 / 08 / 10$ & $2016 / 08 / 31$ & $2017 / 07 / 16$ & \\
\hline & $2012 / 08 / 06$ & $2013 / 08 / 10$ & $2014 / 09 / 15$ & $2015 / 08 / 21$ & $2016 / 09 / 19$ & $2017 / 07 / 24$ & \\
\hline & $2012 / 08 / 17$ & $2013 / 08 / 20$ & $2014 / 10 / 26$ & $2015 / 08 / 31$ & $2016 / 09 / 29$ & $2017 / 08 / 04$ & \\
\hline & $2012 / 08 / 26$ & $2013 / 08 / 30$ & & $2015 / 09 / 10$ & & $2017 / 08 / 14$ & \\
\hline & 2012/09/07 & $2013 / 09 / 12$ & & $2015 / 09 / 18$ & & $2017 / 08 / 23$ & \\
\hline & $2012 / 09 / 19$ & $2013 / 09 / 21$ & & $2015 / 09 / 28$ & & $2017 / 09 / 06$ & \\
\hline & $2012 / 09 / 30$ & $2013 / 09 / 30$ & & & & $2017 / 09 / 17$ & \\
\hline & $2012 / 10 / 13$ & $2013 / 10 / 11$ & & & & $2017 / 09 / 27$ & \\
\hline Tara NESW (\#31) & 2005 & 2006 & 2007 & 2008 & 2009 & 2010 & 2011 \\
\hline & - & - & $2007 / 08 / 02$ & $2008 / 07 / 08$ & $2009 / 07 / 09$ & $2010 / 07 / 09$ & $2011 / 07 / 22$ \\
\hline & & & $2007 / 08 / 16$ & $2008 / 07 / 16$ & $2009 / 07 / 17$ & $2010 / 08 / 09$ & $2011 / 08 / 09$ \\
\hline & & & & $2008 / 07 / 23$ & $2009 / 07 / 29$ & & 2011/08/19 \\
\hline & & & & $2008 / 07 / 26$ & $2009 / 08 / 12$ & & \\
\hline & & & & $2008 / 08 / 11$ & $2009 / 08 / 15$ & & \\
\hline & & & & $2008 / 08 / 18$ & $2009 / 08 / 28$ & & \\
\hline & 2012 & 2013 & 2014 & 2015 & 2016 & 2017 & \\
\hline & $2012 / 09 / 07$ & $2013 / 06 / 05$ & $2014 / 08 / 16$ & $2015 / 07 / 09$ & $2016 / 07 / 03$ & $2017 / 06 / 18$ & \\
\hline & $2012 / 09 / 18$ & $2013 / 07 / 06$ & $2014 / 08 / 28$ & $2015 / 07 / 20$ & $2016 / 07 / 20$ & $2017 / 06 / 28$ & \\
\hline & $2012 / 09 / 29$ & 2013/07/18 & 2014/09/06 & $2015 / 07 / 30$ & $2016 / 07 / 29$ & $2017 / 07 / 05$ & \\
\hline & & $2013 / 08 / 01$ & $2014 / 09 / 15$ & $2015 / 08 / 10$ & $2016 / 08 / 10$ & $2017 / 07 / 16$ & \\
\hline & & $2013 / 08 / 20$ & $2014 / 09 / 26$ & $2015 / 08 / 21$ & $2016 / 08 / 31$ & $2017 / 07 / 24$ & \\
\hline & & 2013/08/30 & 2014/10/07 & $2015 / 08 / 31$ & 2016/09/19 & 2017/08/04 & \\
\hline & & $2013 / 09 / 12$ & 2014/10/17 & 2015/09/10 & $2016 / 09 / 29$ & 2017/08/14 & \\
\hline & & $2013 / 09 / 21$ & & $2015 / 09 / 18$ & & $2017 / 08 / 23$ & \\
\hline & & $2013 / 09 / 30$ & & $2015 / 09 / 28$ & & 2017/09/06 & \\
\hline & & & & & & $2017 / 09 / 17$ & \\
\hline & & & & & & $2017 / 09 / 27$ & \\
\hline
\end{tabular}




\begin{tabular}{|c|c|c|c|c|c|c|c|}
\hline & & & & & & $2017 / 10 / 07$ & \\
\hline \multirow[t]{4}{*}{ Tara NWSE (\#31) } & 2005 & 2006 & 2007 & 2008 & 2009 & 2010 & 2011 \\
\hline & - & - & - & $\begin{array}{l}2008 / 08 / 18 \\
2008 / 08 / 25 \\
2008 / 09 / 05\end{array}$ & $2009 / 08 / 28$ & - & - \\
\hline & 2012 & 2013 & 2014 & 2015 & 2016 & 2017 & \\
\hline & - & - & - & - & - & - & \\
\hline \multirow[t]{4}{*}{ Eguerit EW (\#40) } & 2005 & 2006 & 2007 & 2008 & 2009 & 2010 & 2011 \\
\hline & - & - & - & $2008 / 08 / 20$ & - & - & - \\
\hline & 2012 & 2013 & 2014 & 2015 & 2016 & 2017 & \\
\hline & - & - & - & - & - & - & - \\
\hline \multirow[t]{4}{*}{ Bilantao NESW (\#41) } & 2005 & 2006 & 2007 & 2008 & 2009 & 2010 & 2011 \\
\hline & - & - & $2007 / 07 / 30$ & $\begin{array}{l}2008 / 08 / 17 \\
2008 / 08 / 26\end{array}$ & $2009 / 09 / 01$ & - & - \\
\hline & 2012 & 2013 & 2014 & 2015 & 2016 & 2017 & \\
\hline & - & 2013/07/13 & - & - & - & - & \\
\hline
\end{tabular}

